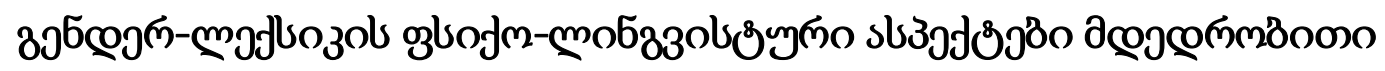

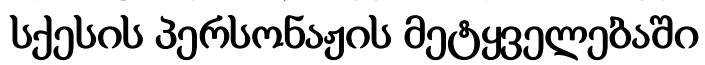

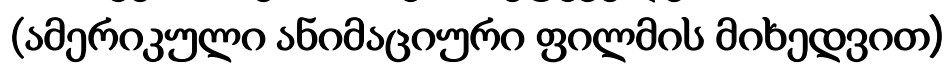

\author{
мsґовి

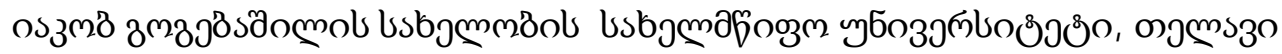

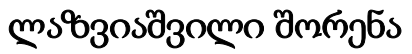

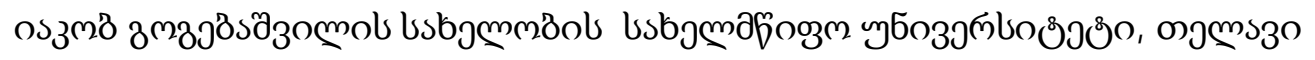

https://doi.org/10.52340/idw.2021.514

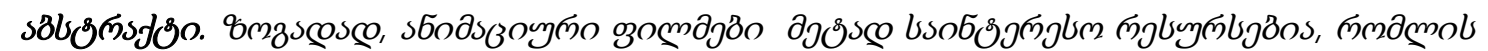

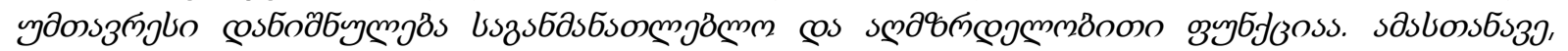

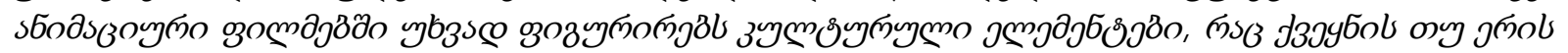

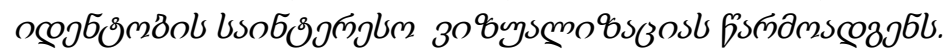

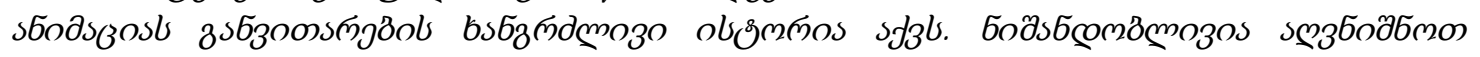

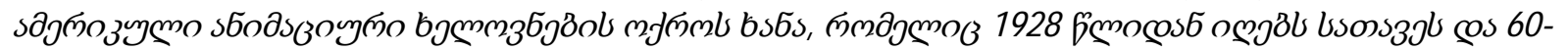

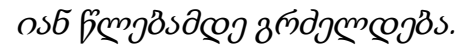

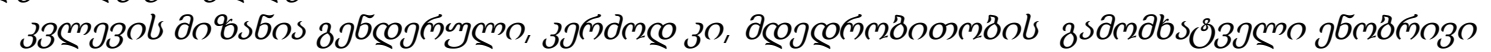

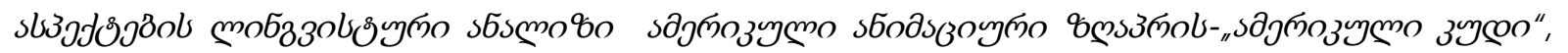

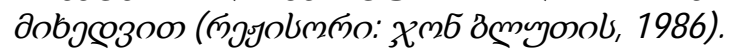

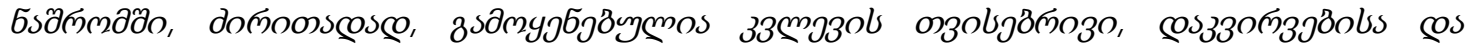

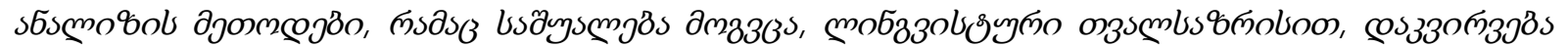

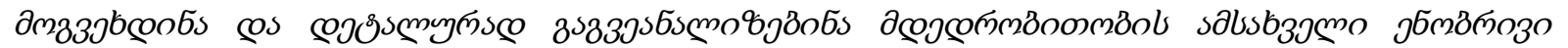

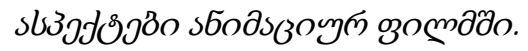

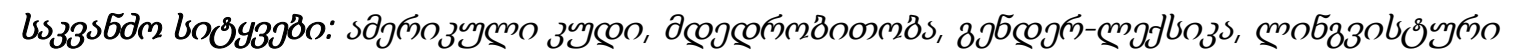

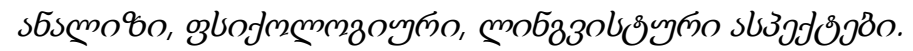

\section{gjuszsmo}

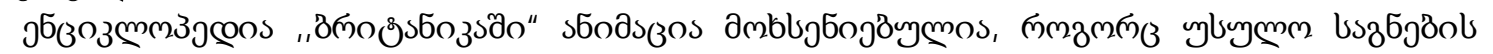

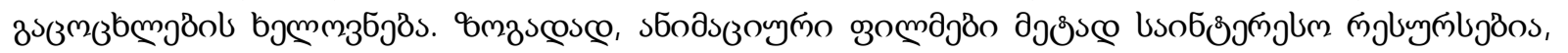

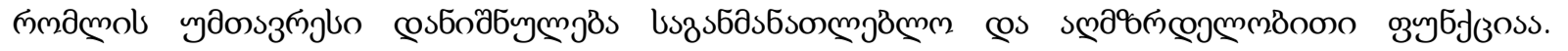

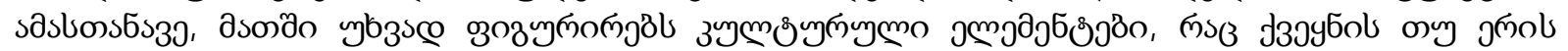

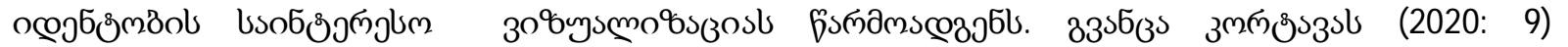

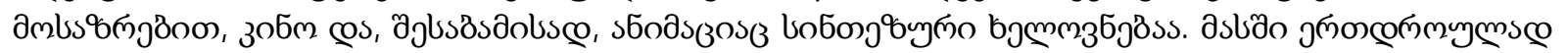

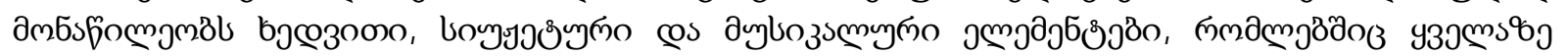
дбодаббјммз зьбо дзоб

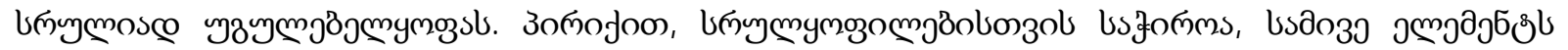

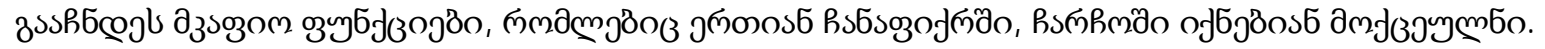

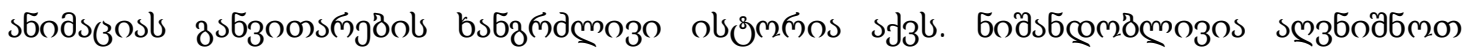

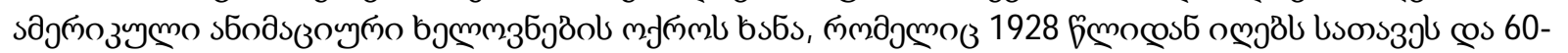

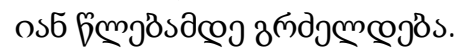

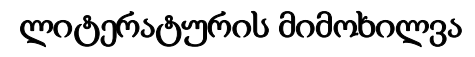




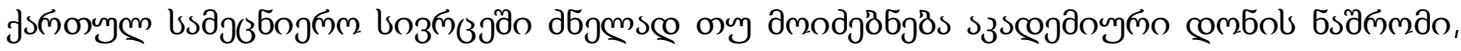

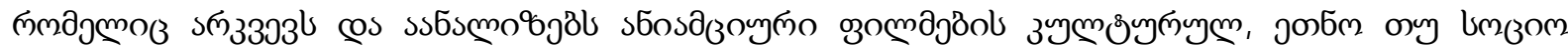

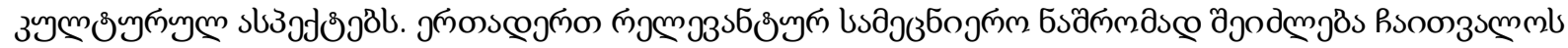

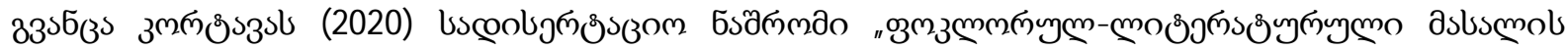

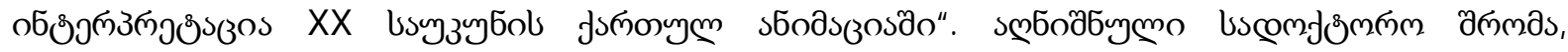

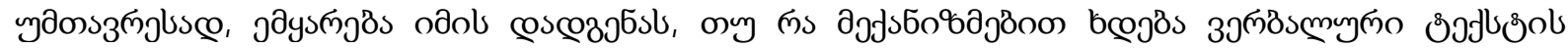

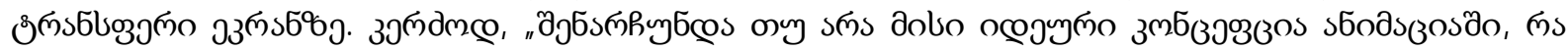

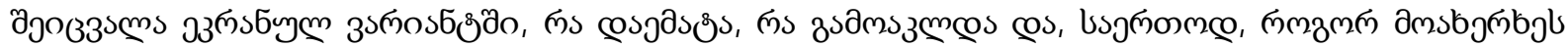

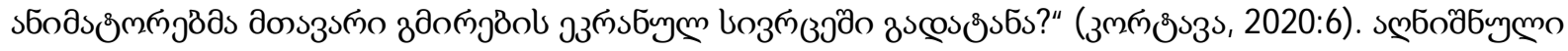

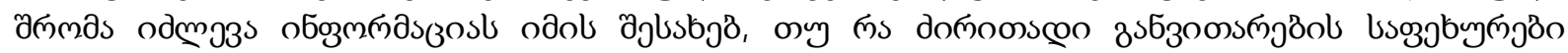

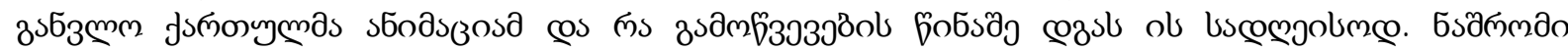

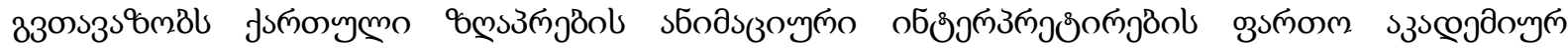

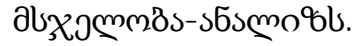

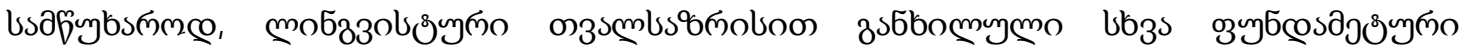

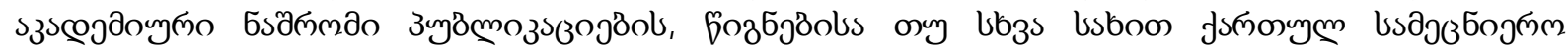

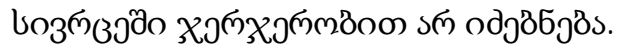

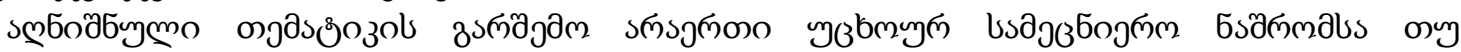

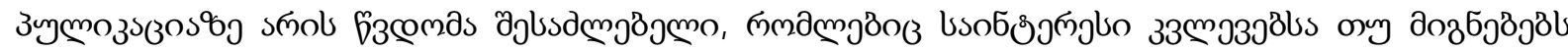

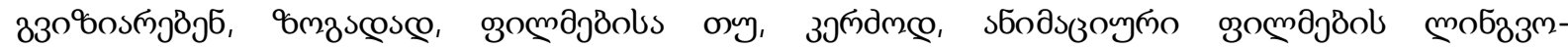

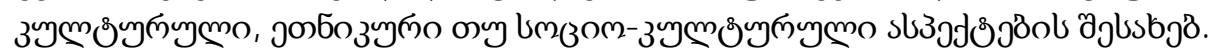

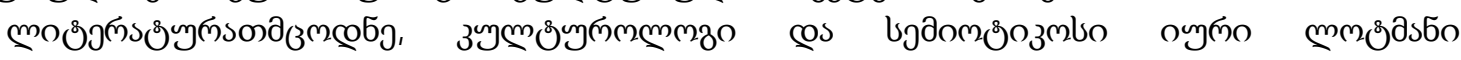

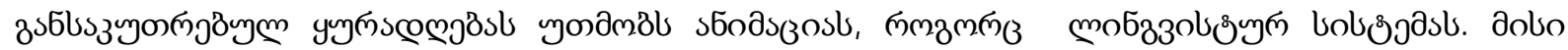

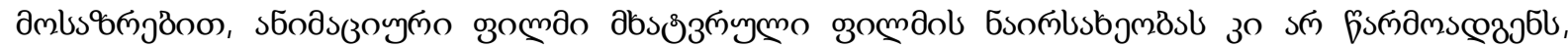

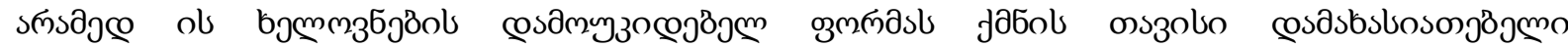

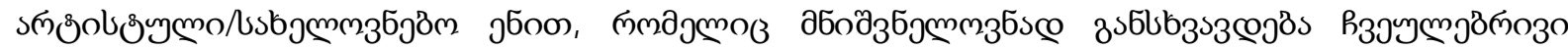

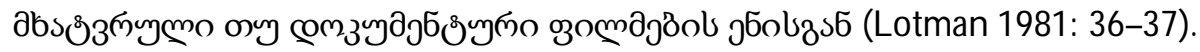

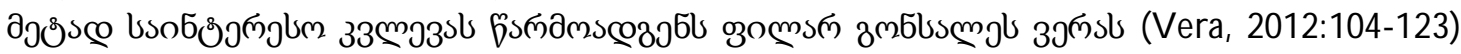

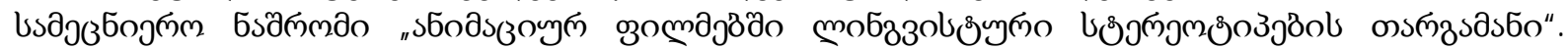

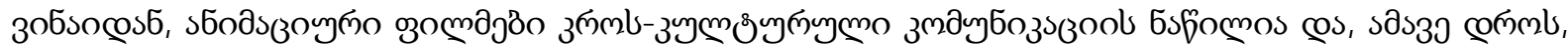

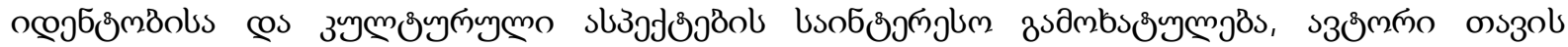

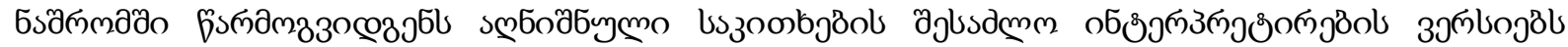

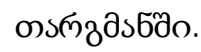

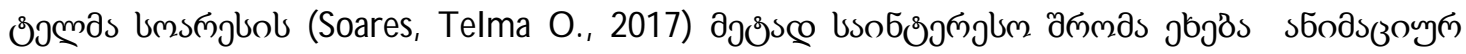

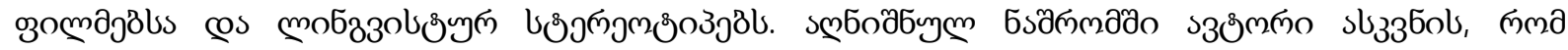

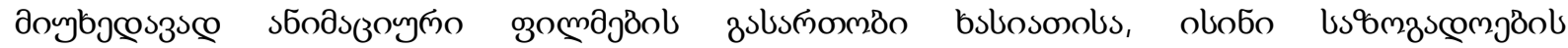

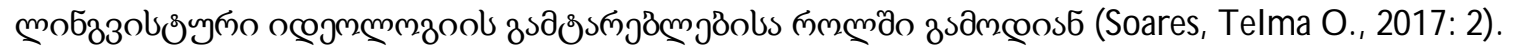

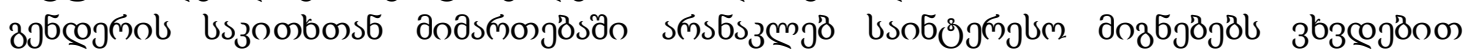
coszoluob (Davis, 2001) bscobj๓

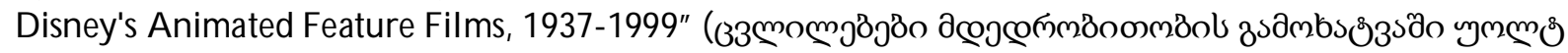

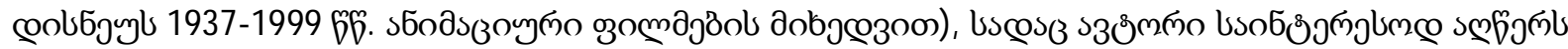

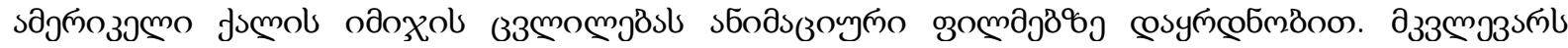

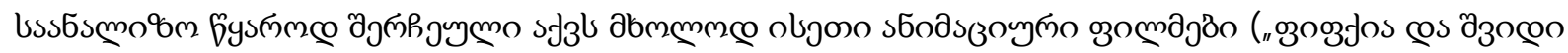

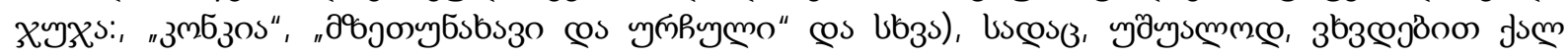

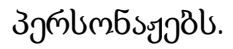

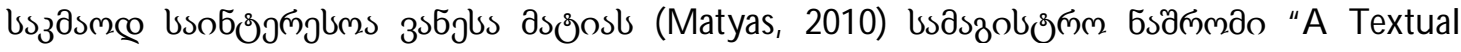

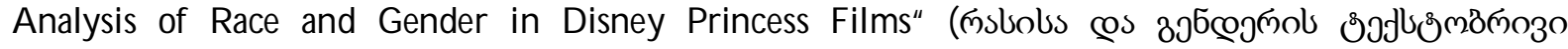

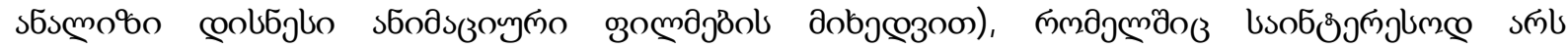

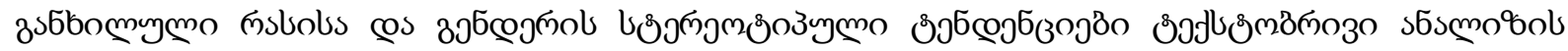
combj\%?. 


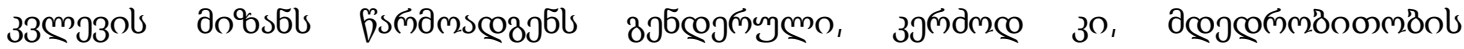

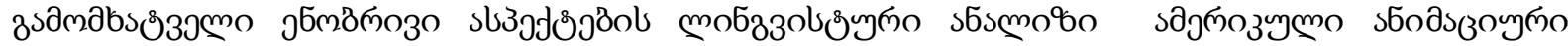

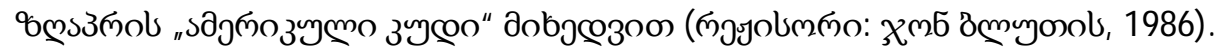

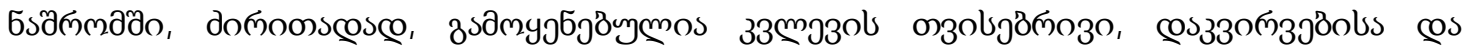

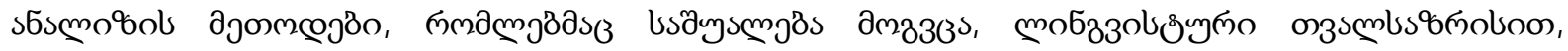

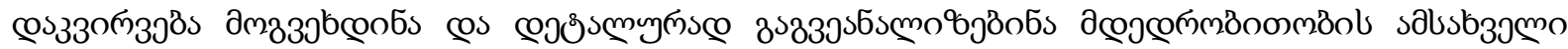

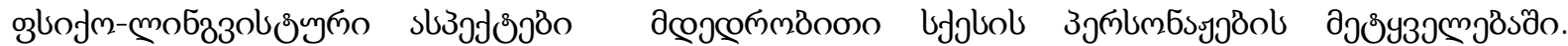

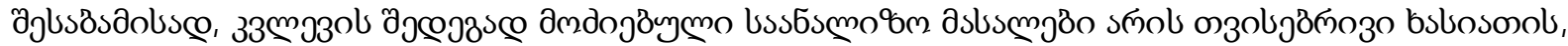

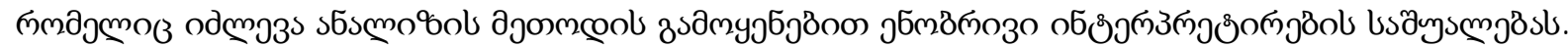

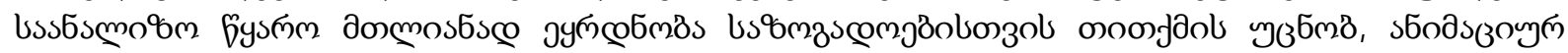

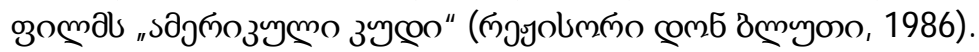

\section{dornooscoo 6sfomo}

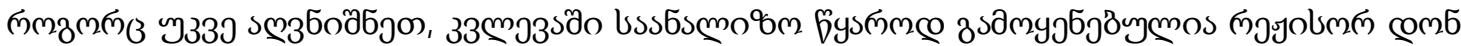

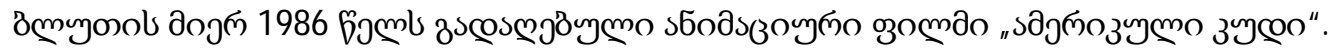

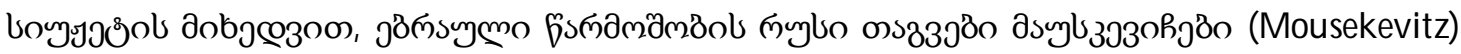

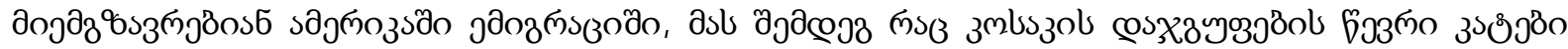

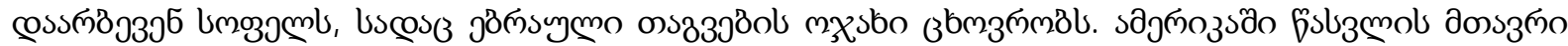

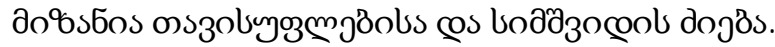

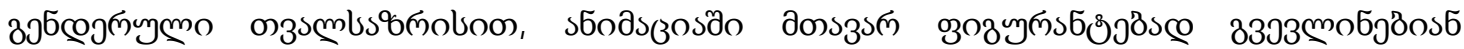

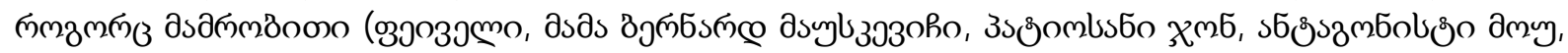

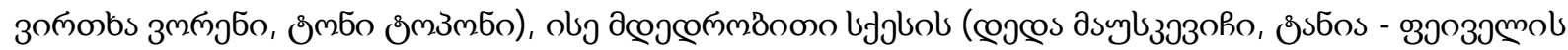

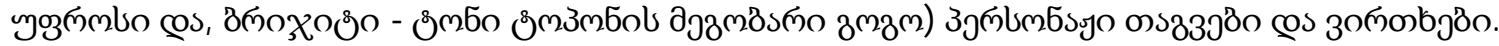

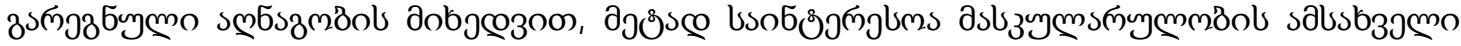

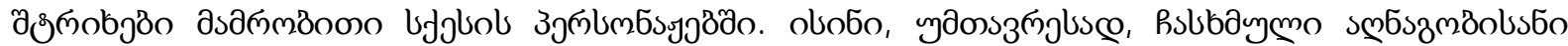

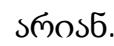
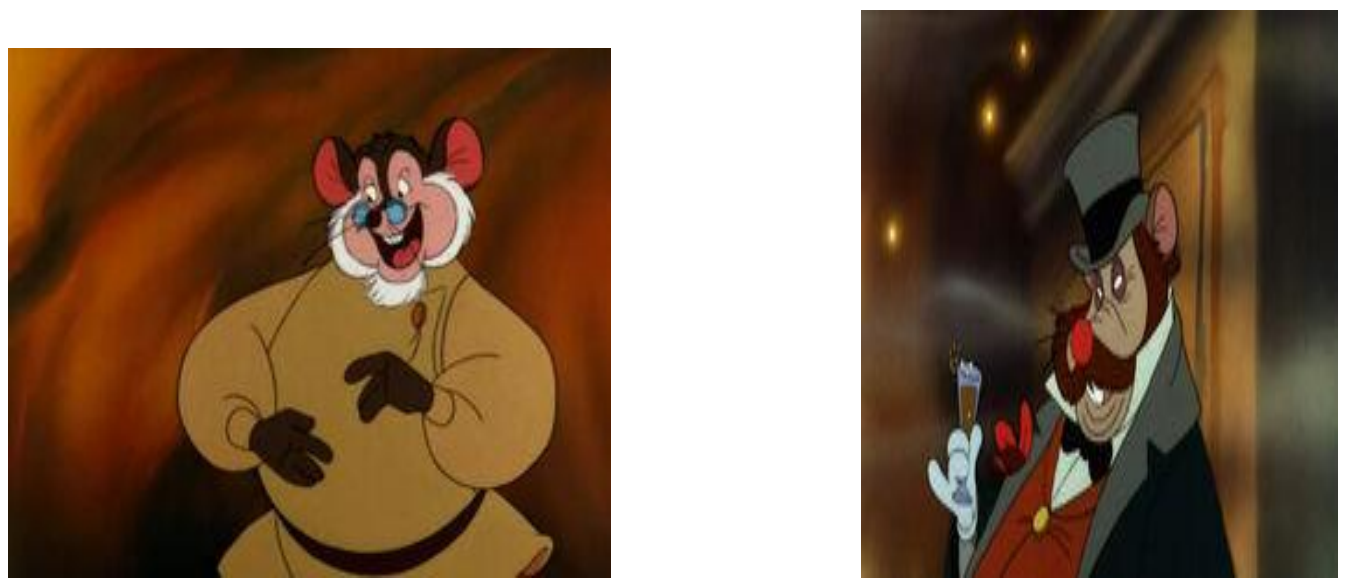

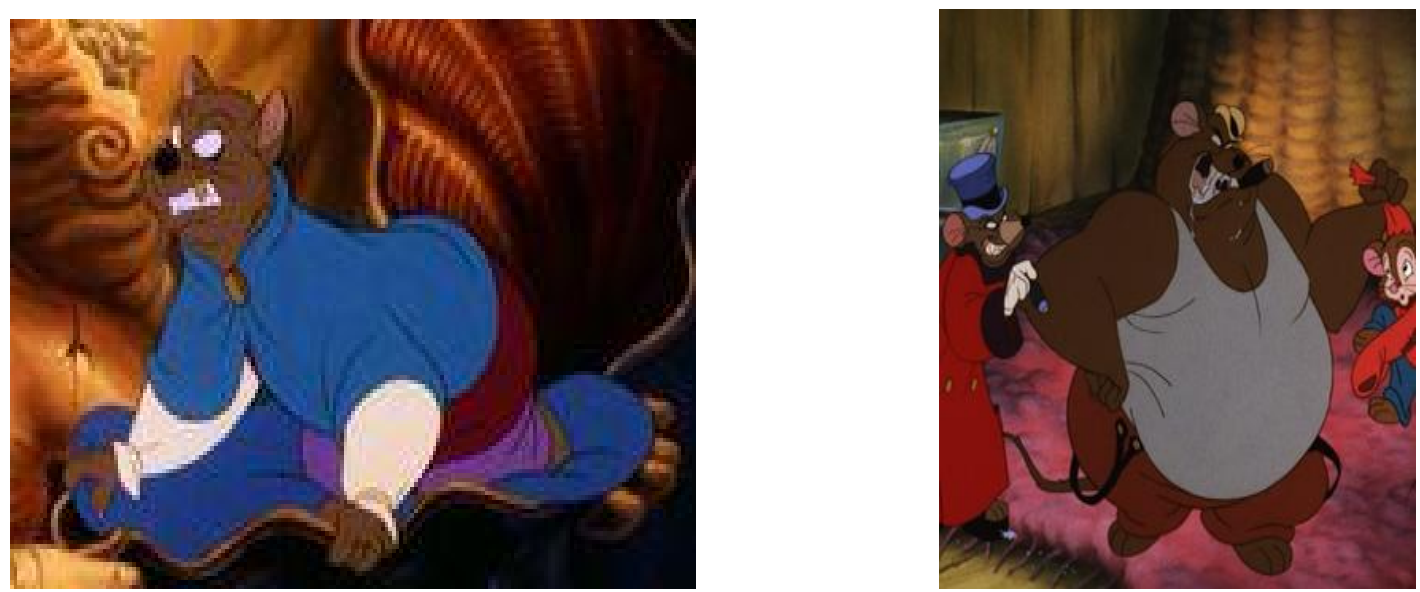

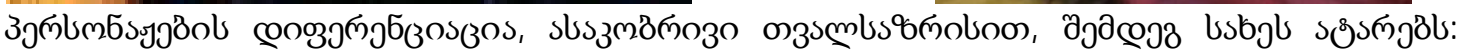

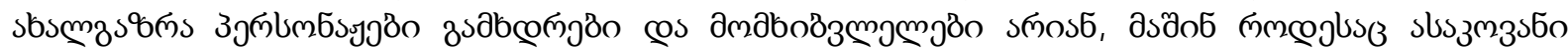

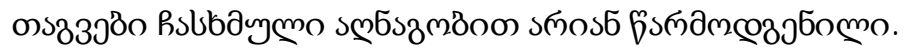

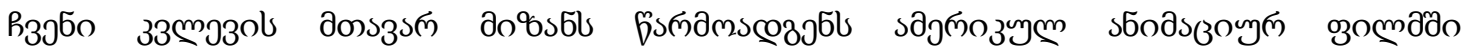

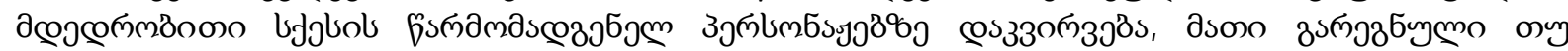

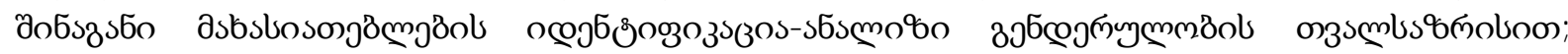

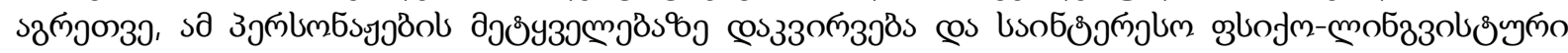

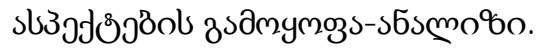

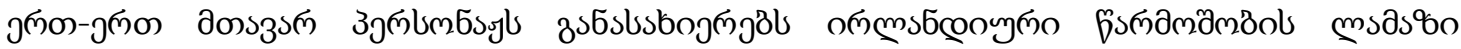

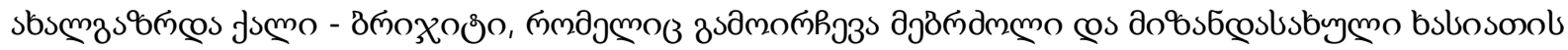

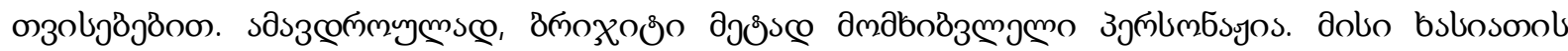

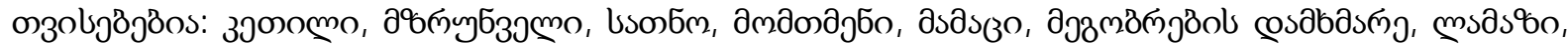

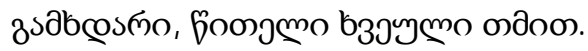

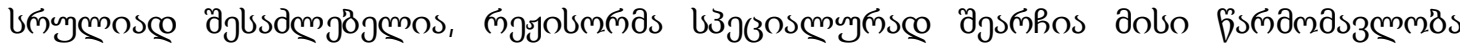

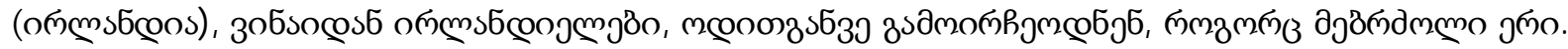

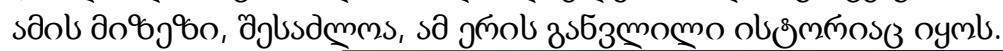

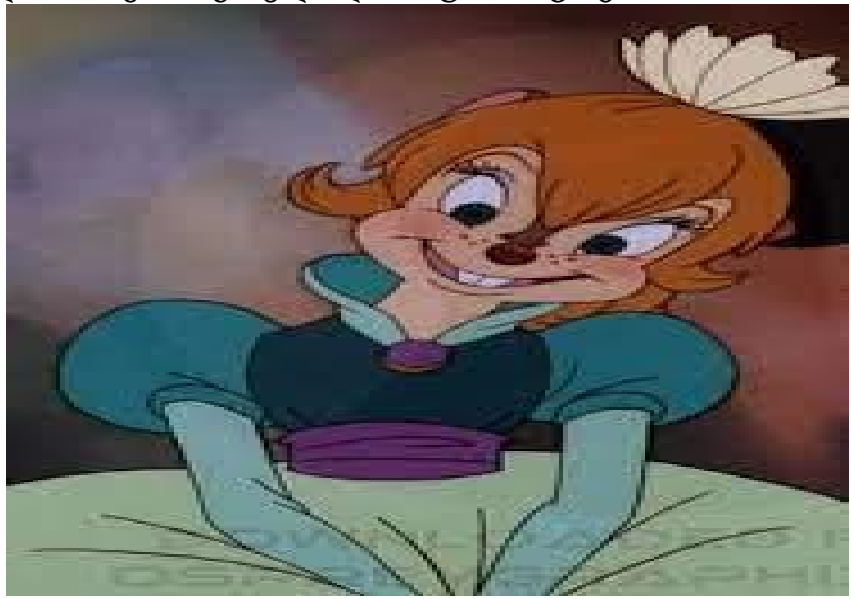

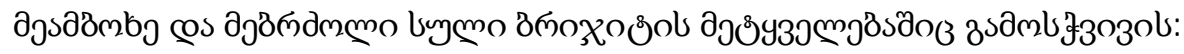

- I ask you this. A re we going to stand by and let cats wreck our homes, our businesses and our lives? If

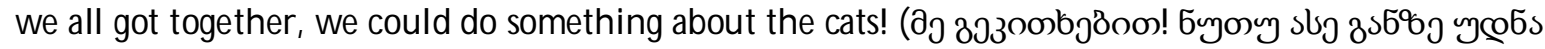

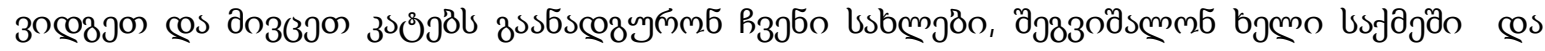

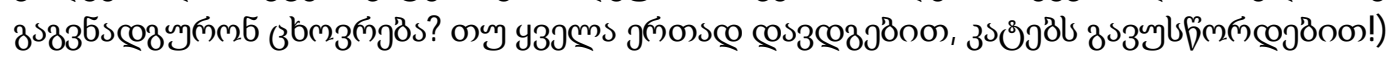

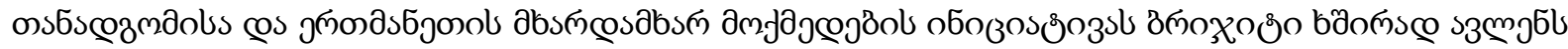

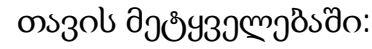


- Wait a minute. Come back! W here are you going? Oh S-so, if we all got together, we could do

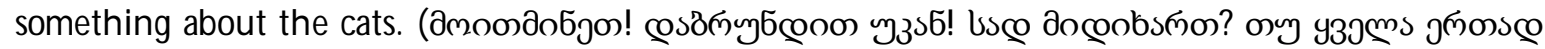

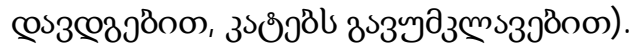

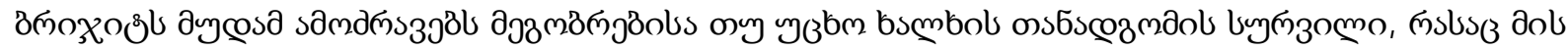

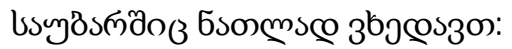

- Oh, Fillie. Honest John, he's lost his family. By any chance, do you know the Mousekewitzes? (my,

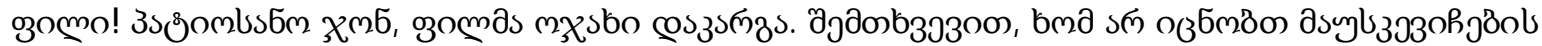
mxsbls?)

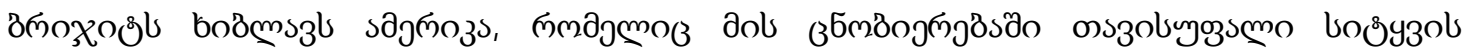

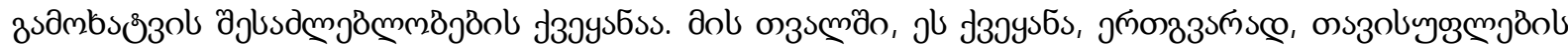

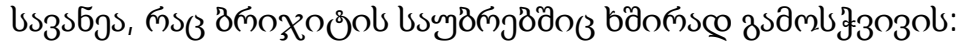

- This is A merica. Don't be afraid. A re you going to let those cats push you around? (ju sajnozss.

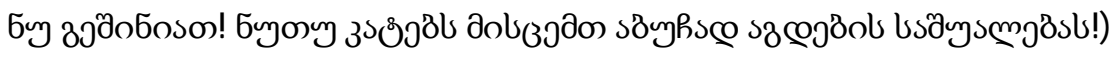

- This is A merica. We have free speech. Cat, cat, cat! And double cat! ( jl sagnоzss. boóyzol

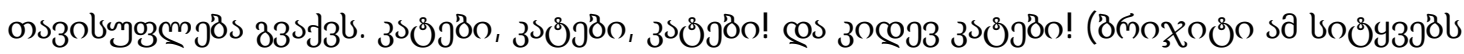

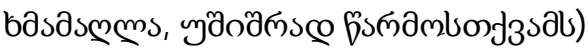

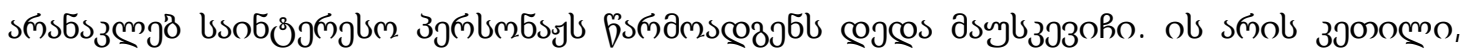

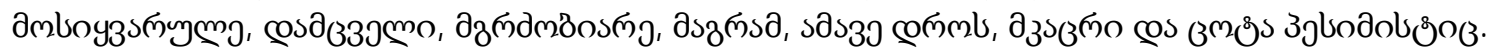

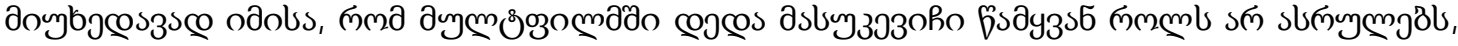

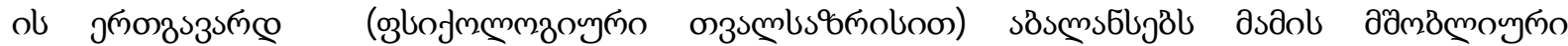

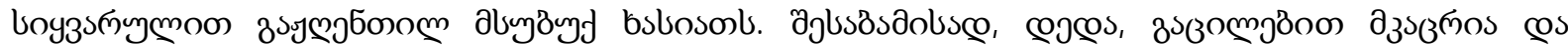

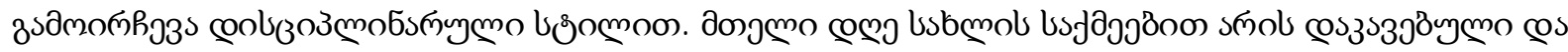

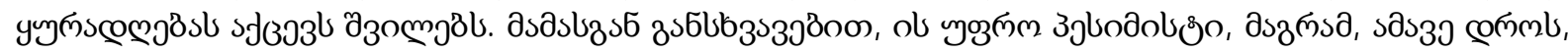

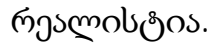

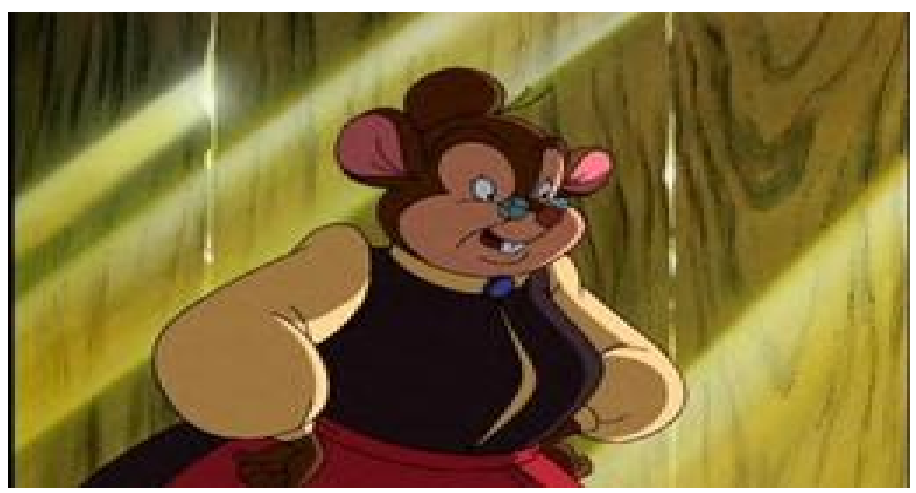

uso6ఠి

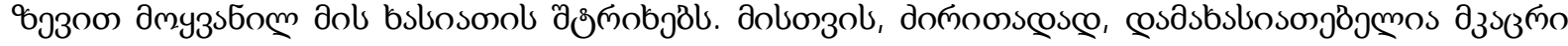

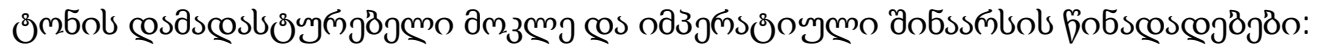

- Tanya, Fievel? W ill you stop that twirling, twirling? Uh, no. No more. That's it. Time for bed.

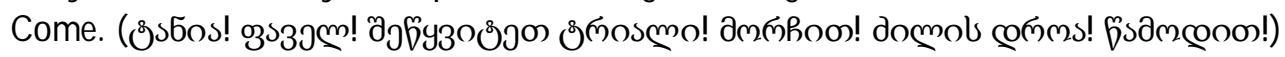

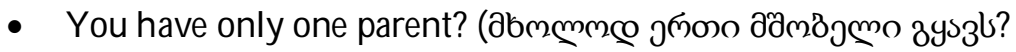

- Shh! Don't say that word. Talk about something else. ( ซø̃! by odsbo sasu! ubzs msajoj obsuzbrg!)

- You're talking nonsense. (bobuymgmgls såm8)

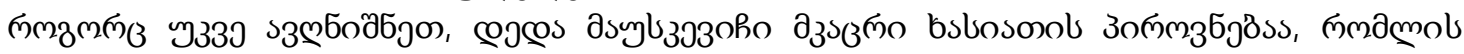

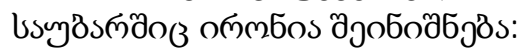

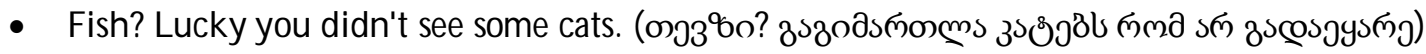

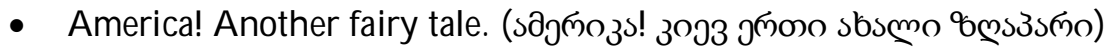




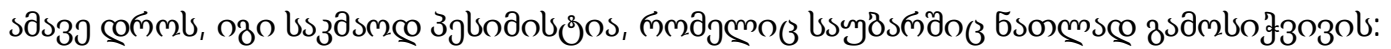

- Oh, it's the end of the world. (m3, gl bma bsaysmmls cosbsbmymos)

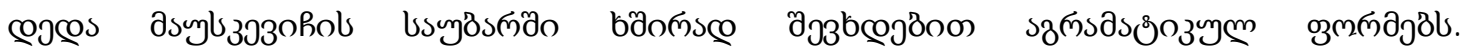

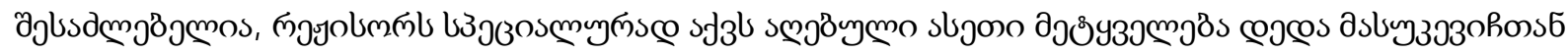

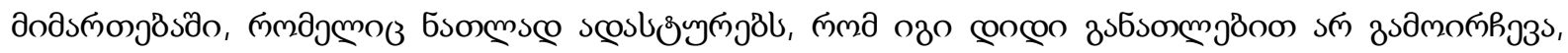

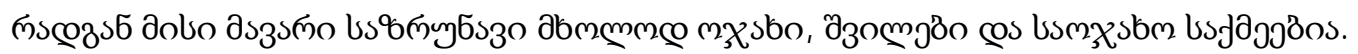

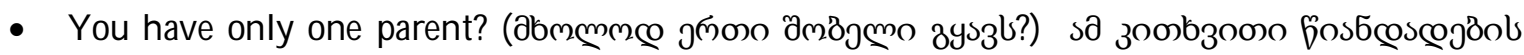

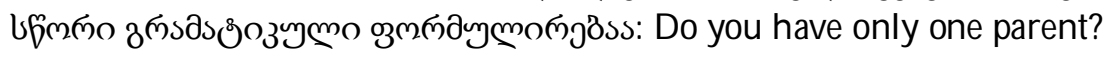

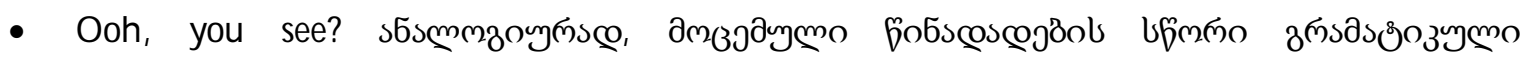

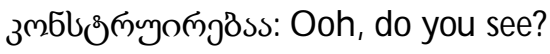

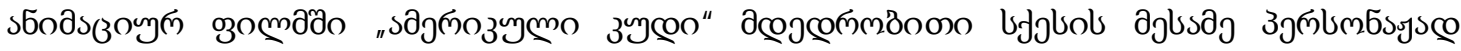

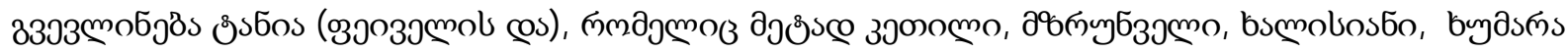
cos msasbo 3jøumbsyos.

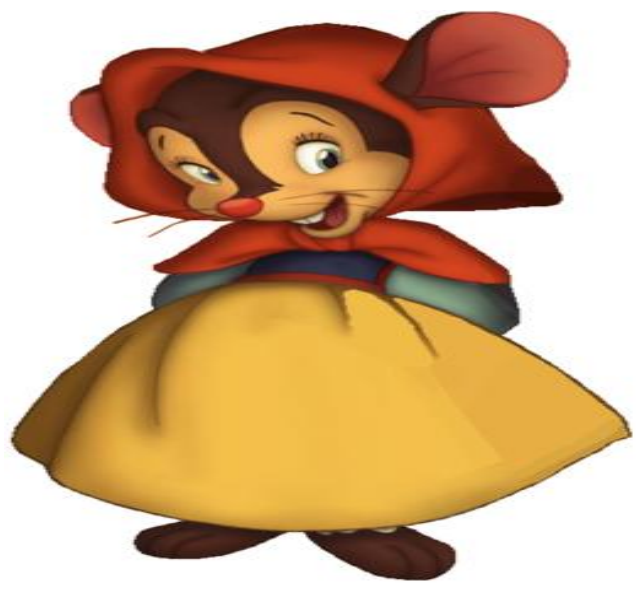

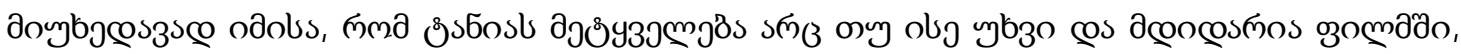

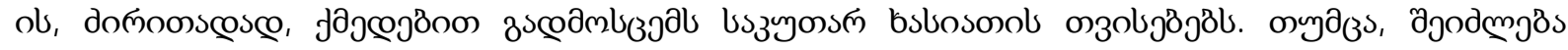

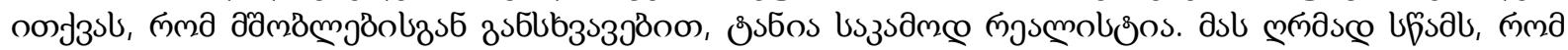

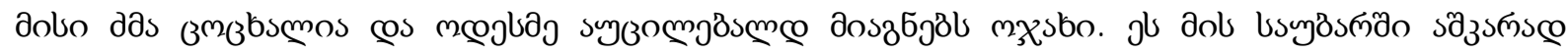
дgoбozбjठs:

- They changed my name to Tilly. Maybe they changed Fievel's name to Fillie. Come on, Papa.

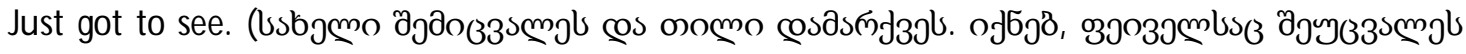

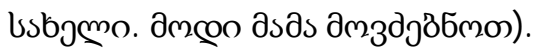

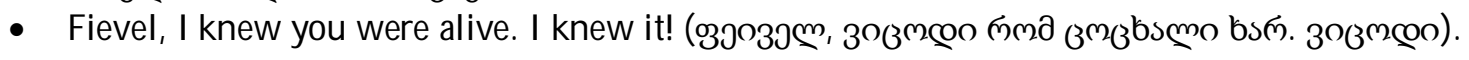

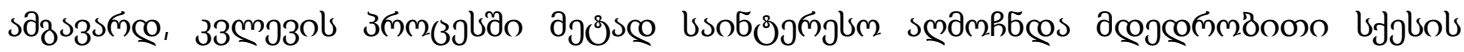

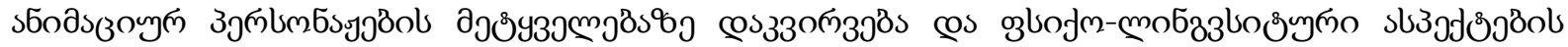

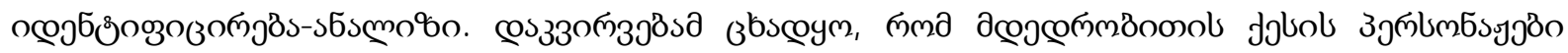

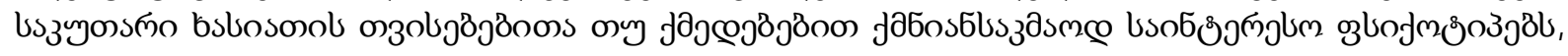

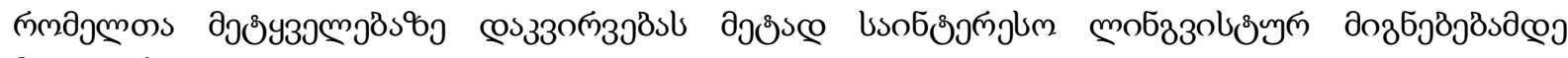
дозуззऽю́о.

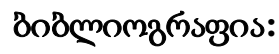

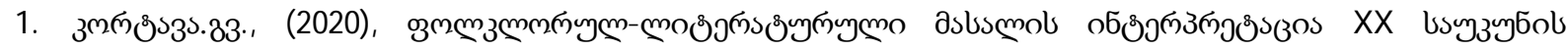

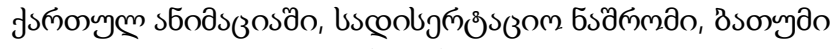

2. Lotman, J., (1976), Semiotics of Cinema. Michigan Slavic Contributions 5. Ann Arbor: University of M ichigan Press. 
3. Vera, P., 92012), The translation of linguistic stereotypes in animated films: a case study of DreamW orks' Shrek and Shark Tale, The Journal of Specialised Translation, I ssue 17 - January 2012, pp.104-123

4. Soares, Telma O.. (2017). Animated Films and Linguistic Stereotypes: A Critical Discourse Analysis of Accent Use in Disney Animated Films. In BSU Master's Teses and Projects. Item 53. A vailable at htp://vc.bridgew .edu/theses/53

5. Davis, AM; (2001) Disney's women: changes in depictions of femininity in W alt Disney's animated feature films, 1937-1999. Doctoral thesis, University of London.

6. Matyas.V., (2010), A Textual A nalysis of Race and Gender in Disney Princess Films, M aster Thesis, M cM aster University

\title{
Psycho-Linguistic Aspects of Gender Vocabulary in Female Characters (Based on the American Animated M ovie)
}

\author{
Garibashvili Manana \\ Iakob Gogebashvili State University, Telavi \\ Lazviashvili Shorena \\ lakob Gogebashvili Telavi State University
}

\begin{abstract}
In general, animated films are very interesting resources carrying the educational and upbringing functions. In addition, animated movies are rich in cultural elements that are an interesting visualization of the identity of a country or a nation.

A nimation has a long history of development. It is notew orthy to mention that the golden age of A merican animated art originated in 1928 and lasted until the 60s.

The aim of the study is to provide a linguistic analysis of the linguistic aspects of gender, in particular femininity based on the American animated movie "American Tail" directed by Don Bluth in 1986 .

The paper mainly uses qualitative research, observation and analysis methods, which allowed us to observe and analyze in detail the psycho-linguistic aspects of femininity in the speech of female characters.
\end{abstract}

Key words: American Tail, animated movie, femininity, gender vocabulary, linguistic analysis, psychological, linguistic aspects. 